\title{
Desafios dos profissionais de enfermagem frente a pandemia do coronavírus sars-
}

\section{$\operatorname{cov} 2$}

\author{
Challenges of nursing professionals in front of the pandemic of the coronavirus sars-cov2
}

Retos de los profesionales de enfermería frente a la pandemia del coronavirus sars-cov2

Recebido: 21/05/2021 | Revisado: 26/05/2021 | Aceito: 27/05/2021 | Publicado: 09/06/2021

\author{
Ana Maria Ferreira do Nascimento \\ ORCID: https://orcid.org/0000-0002-6967-8114 \\ Centro Universitário Santo Agostinho, Brasil \\ E-mail: anamariaferreranasc1@gmail.com \\ Isamara Ferreira Souza Rêgo \\ ORCID: https://orcid.org/ 0000-0002-0770-3222 \\ Centro Universitário Santo Agostinho, Brasil \\ E-mail:isa.mmaarraa@gmail.com \\ Magda Rogéria Pereira Viana \\ ORCID: https:// orcid.org/0000-0003-3293-7095 \\ Centro Universitário Santo Agostinho, Brasil \\ E-mail:magdarogeria@hhotmail.com
}

\begin{abstract}
Resumo
O novo Coronavírus (COVID-19) constitui uma Emergência de Saúde Pública de Importância Internacional - o mais alto nível de alerta da Organização Mundial de Saúde. Os profissionais da enfermagem têm enfrentado rotineiramente precarização no processo de trabalho e inúmeros problemas no sistema de saúde. O objetivo desse estudo foi objetivo analisar as evidências das publicações científicas acerca dos desafios dos profissionais de enfermagem frente à pandemia do Coronavírus SARS-COV2. Utilizou-se a revisão integrativa, em que 10 artigos científicos disponibilizados na íntegra, nos últimos 5 anos, selecionados na Biblioteca Virtual de Saúde, compuseram a amostra analisada. Os resultados evidenciaram os desafios enfrentados pela enfermagem frente o contexto pandêmico em questão. Mediante a isso pode-se concluir que os profissionais de enfermagem sempre foram desafiados ao longo do tempo e com a chegada da pandemia pelo Coronavírus SARS-COV2 esses desafios tomaram proporções inimagináveis.
\end{abstract}

Palavras-chave: Covid-19; Infeção; Enfrentamento de enfermagem.

\begin{abstract}
The new Coronavirus (COVID-19) constitutes a Public Health Emergency of International Importance - the highest level of alert by the World Health Organization. Nursing professionals have routinely faced precariousness in the work process and numerous problems in the health system. The aim of this study was to analyze the evidence from scientific publications about the challenges faced by nursing professionals in the face of the SARS-COV2 Coronavirus pandemic. The integrative review was used, in which 10 scientific articles made available in full, in the last 5 years, selected in the Virtual Health Library, comprised the analyzed sample. The results showed the challenges faced by nursing in the context of the pandemic context in question. As a result, it can be concluded that nursing professionals have always been challenged over time and with the arrival of the pandemic by the SARSCOV2 Coronavirus these challenges have taken on unimaginable proportions.
\end{abstract}

Keywords: Covid-19; Infection; Coping nursing.

\section{Resumen}

El nuevo Coronavirus (COVID-19) constituye una Emergencia de Salud Pública de Importancia Internacional - el más alto nivel de alerta de la Organización Mundial de la Salud. Los profesionales de enfermería se han enfrentado de manera rutinaria a la precariedad en el proceso de trabajo y numerosos problemas en el sistema de salud. El objetivo de este estudio fue analizar la evidencia de publicaciones científicas sobre los desafíos que enfrentan los profesionales de enfermería frente a la pandemia de coronavirus SARS-COV2. Se utilizó la revisión integradora, en la que 10 artículos científicos puestos a disposición en su totalidad, en los últimos 5 años, seleccionados en la Biblioteca Virtual en Salud, conformaron la muestra analizada. Los resultados mostraron los desafíos que enfrenta la enfermería en el contexto del contexto pandémico en cuestión. Como resultado, se puede concluir que los profesionales de enfermería siempre han sido desafiados con el tiempo y con la llegada de la pandemia por el coronavirus SARS-COV2 estos desafíos han adquirido proporciones inimaginables.

Palabras clave: Covid-19; Infección; Afrontamiento de enfermería. 


\section{Introdução}

A COVID-19 desencadeada pelo vírus denominado como o novo coronavírus, uma doença de contaminação rápida, que é transmitida de uma pessoa para a outra pelas vias respiratórias teve a sua primeira disseminação por toda a China, em seguida sendo espalhada por mais de 200 países. No dia 30 de janeiro de 2020 após os estragos já visualizados pela Organização Mundial de Saúde (OMS) fez com que esta declarasse Emergência de Saúde Pública de Importância Internacional, e somente no mês de março, mais especificamente o dia 11 a COVID-19 foi denominada como pandemia (Marques et al., 2020).

Por se tratar de uma doença nova torna-se visível a falta de recursos materiais principalmente no sistema único de saúde (SUS), com isso os profissionais de saúde que estão na linha de frente em especial o profissional enfermeiro deparam-se com situações e condições precárias no âmbito de atuação, além da escassez de Equipamentos de proteção individual (EPI's) o que os torna mais propensos à contaminação. No que se refere a transmissão, a mesma ocorre principalmente através de gotículas respiratórias, em procedimentos que coloquem as vias respiratórias do indivíduo em contato com algum possível transmissor, como aerossóis e intubação orotraqueal e contato com objetos contaminados. Vale ressaltar que os indivíduos tanto sintomáticos como assintomáticos transmitem o vírus por conta da sua alta transmissibilidade (Quadro et al., 2020).

Devido as condições precárias de trabalho segundo o Conselho Federal de Enfermagem (COFEN) o número de profissionais de enfermagem contaminados e/ou mortos em decorrência da Covid-19 cresce de maneira assustadora em todo o território mundial, em meados de 2020 o número de contaminados chegou a 18 mil, já as mortes passavam de 200. É sabido que a enfermagem tem papel essencial na assistência e combate à pandemia, ficando mais evidente a sua indispensabilidade na linha de frente no combate a Covid-19 (Santos et al., 2020).

O profissional enfermeiro já enfrenta diversos desafios diariamente nas suas atividades de trabalho, e diante do enfrentamento à Covid-19 os desafios são muitos principalmente no que se refere a precariedade e problemas existentes no sistema de saúde, já citados anteriormente, destacando-se as longas jornadas de trabalho lado a lado com salários desproporcionais a essa jornada (Quadros et al., 2020).

Vale lembrar que muitos desses problemas enfrentados pelas equipes de saúde já eram vivenciados, apenas foram exacerbados com o aparecimento da Covid-19. Além disso verificado o cenário atual do Brasil, muitos fatores contribuem com os adoecimentos dos profissionais (Quadros et al., 2020). Os profissionais de enfermagem representam 60\% da mão de obra brasileira dos trabalhadores da saúde, em que os enfermeiros chegam a mais de 550 mil desses profissionais, os técnicos de enfermagem representam 1,3 milhão e os auxiliares de enfermagem chegam a 400 mil (Santos et al.,2020).

Atuando na linha de frente da pandemia ao do Coronavírus, oito em cada dez da mão de obra trabalhista dos profissionais de enfermagem são representados pelo sexo feminino, sendo que boa parte dessas mulheres também assumem o papel de provedora da família, tornando-se desafiador o retorno para casa após a jornada laboral no convívio com a presença do vírus no ambiente de trabalho, pois em sua residência, ainda cuidam dos filhos, além de dar assistência aos idosos e doentes dentro dos seus ambientes familiares (Santos et al.,2020).

Há uma desproporcionalidade com relação às categorias profissionais que estão na frente do enfrentamento à pandemia pelo COVID-19, sendo que a enfermagem estará sempre mais exposta ao contágio pelo vírus, visto que essa categoria presta a maioria dos cuidados diretos aos infectados e permanecem longos períodos ao lado dos pacientes e isso torna-os mais propícios ao contágio do Coronavírus SARS-COV2 (MINAYO e FREIRE, 2020).

Atualmente, vivencia-se uma grande crise mundial, sendo que há uma enorme instabilidade relacionada a saúde dos profissionais de enfermagem mediante a presença do Vírus no ambiente de trabalho, que por muitas vezes, têm que dar o 
melhor de si em condições de trabalho inadequadas, objetivando a assistência aos pacientes, assim como também não deixar a população à mercê da sorte (Soares et al.,2020).

No período de 5 de abril a 15 de abril de 2020, houve no Brasil um crescimento significativo de 18 vezes no número de profissionais de enfermagem confirmados ou com suspeita de infecção pelo vírus SARS-COV2, tornando-se um número contínuo e crescente de profissionais infectados (Quadros et al.,2020).

O Cofen tem registrado várias denúncias relacionadas à escassez de recursos materiais, a falta de mão de obra tecnicamente treinada, deficiência na testagem, inadequação nos locais de trabalho, subdimencionamento, além disso a um número alto de profissionais afastados por fazerem parte do grupo de risco, gerado a necessidade da mão de obra de mais profissionais para compensar a ausência destes, e isso tem deixado esse grupo mais exposta ao adoecimento (Quadros et al.,2020).

O Conselho dos profissionais de enfermagem tem demonstrado muita preocupação com a categoria, pois entende que proteger a saúde destes profissionais é proteger a saúde do Brasil, visto que os mesmos precisam estar bem para que possam cuidar da população brasileira (Santos et al., 2020).

Consequentemente, à essa situação vivenciada pelos profissionais de enfermagem foi iniciado pelo Cofen, medidas institucionais com vistas aos desafios enfrentados pela enfermagem durante a pandemia pela COVID-19 para apoiar todas as equipes de enfermagem que se encontram em uma batalha diária no combate ao vírus SARS-COV2 (Santos et al., 2020). Este estudo tem como objetivo averiguar as publicações científicas acerca dos desafios dos profissionais de enfermagem frente à pandemia do Coronavírus SARS-COV2.

\section{Metodologia}

O estudo em questão trata-se de uma revisão integrativa de literatura com abordagem qualitativa para embasar análises de estudos de pesquisas preponderantes, além de servir de suporte para produções científicas atuais e futuras. Este método tem como objetivo condensar resultados colhidos em estudos que abordem o tema estudado de forma relevante para a pesquisa desenvolvida (Ercole et al., 2021).

O estudo foi desenvolvido a partir do problema de pesquisa, onde para construir o mesmo utilizou-se a estratégia PICo (P: População; I: Interesse; Co: Contexto) que resultou no seguinte problema de pesquisa: Quais as evidências científicas acerca dos desafios dos profissionais de enfermagem frente a pandemia pelo Coronavírus SARS-COV2?

A coleta de dados foi executada entre agosto de 2020 à Maio de 2021, foi feito um levantamento das produções científicas sobre o tema: As evidências científicas acerca dos desafios dos profissionais de enfermagem frente a pandemia pelo Coronavírus SARS-COV2, utilizando-se descritores instituídos pelos Descritores em Saúde (DeCS): Infecção OR Coronavírus OR Covid-19, Coronavírus OR Covid-19 OR Pandemia, Enfermagem OR Assistência OR Coronavírus, Enfermagem OR Enfrentamento $O R$ Pandemia, Enfermagem $O R$ Desafios $O R$ Pandemia, suas correspondentes em inglês por meio da Medical Subject Heading ( MeSH): Infection OR Coronavirus OR Covid-19, Coronavirus OR Covid-19 OR Pandemic, Nursing OR Assistence OR Coronavirus, Nursing OR Coping OR Pandemic, Nursing OR Challenges OR Pandemic.

O levantamento bibliográfico foi realizado por meio de publicações selecionadas pela internet, no endereço eletrônico da plataforma da Biblioteca Virtual de saúde (BVS), ressaltando que os artigos indexados nas seguintes bases de dados: Sistema Online de Busca e Análise de Literatura Médica (MEDLINE) PubMed, Literatura Latino-Americana e do Caribe em Ciências da Saúde ( LILACS), Bases de Dados de Enfermagem ( BDENF) e Scidenific Eletronic Library Online ( Scielo).onde localizou-se artigos nas seguintes bases de dados: LILACS, BDENF, MedLine e Scielo. A análise dos dados referentes à esta pesquisa é de caráter descritivo, onde sumariamente consta as informações sobre os dados contidos na pesquisa. 
Utilizou- se como critério de inclusão os artigos que focassem sobre a temática: desafios dos profissionais de enfermagem frente à pandemia do Coronavírus SARS-COV2 no Brasil, artigos que comtemplassem sobre o trabalho dos profissionais de enfermagem no enfrentamento da Covid-19, artigos na língua portuguesa, inglesa e espanhola que abordassem o tema proposto independentemente do método de pesquisa, artigos descritos na íntegra e gratuitos, publicados nos anos de 2020 à 2021, e como critério de exclusão optou-se por não utilizar artigos que não abordassem sobre os desafios dos profissionais de enfermagem brasileiros frente a pandemia pelo Coronavírus SARS-COV2, textos que se encontravam incompletos, que não estavam disponíveis na íntegra e online, artigos duplicados, dissertações, teses e artigos que não forneciam informações suficientes para a temática em foco determinada pelas pesquisadoras.

Para análise dos dados adotou-se cinco etapas: seleção da pergunta da pesquisa; pesquisa bibliográfica; definição dos critérios de inclusão e exclusão dos estudos; seleção da amostra e análise crítica dos achados. Iniciou-se com a leitura analítica objetivando organizar e resumir as informações encontradas nas fontes pesquisadas no intuído de obter a resposta para o problema de pesquisa.

Os dados contidos nos estudos selecionados foram levantados pelas pesquisadoras, inicialmente através da montagem do fluxograma do prisma para mostra dos resultados, e posteriormente foi elaborada um formulário próprio para caracterização das publicações, em seguida foi realizada a discussão dos resultados através de uma redação descrita.

\section{Resultados}

Inicialmente, por meio de buscas via BVS foram encontrados 117 artigos em bases de dados distintas. Na sequência, foi feita a leitura de 51 artigos únicos onde seus títulos e resumos foram lidos de forma independente por dois revisores. Com base nos critérios de elegibilidade, 25 publicações foram excluídas restando 26 artigos que foram lidos na íntegra por dois revisores independentes, 16 artigos completos foram excluídos, por serem 7 deles duplicados e 9 não eram estudos originais, outros idiomas, não tinha como foco a temática: Desafios dos profissionais de enfermagem frente a pandemia do coronavírus sarscov2, dos estudos incluídos nesta revisão é de 10 artigos. A figura 1 apresenta uma síntese da busca efetuada nas bases de dados, constando informações quanto aos critérios de inclusão e exclusão, artigos selecionados para leitura e artigos incluídos após a leitura. 
Figura 1. Síntese da busca efetuada nas bases de dados.

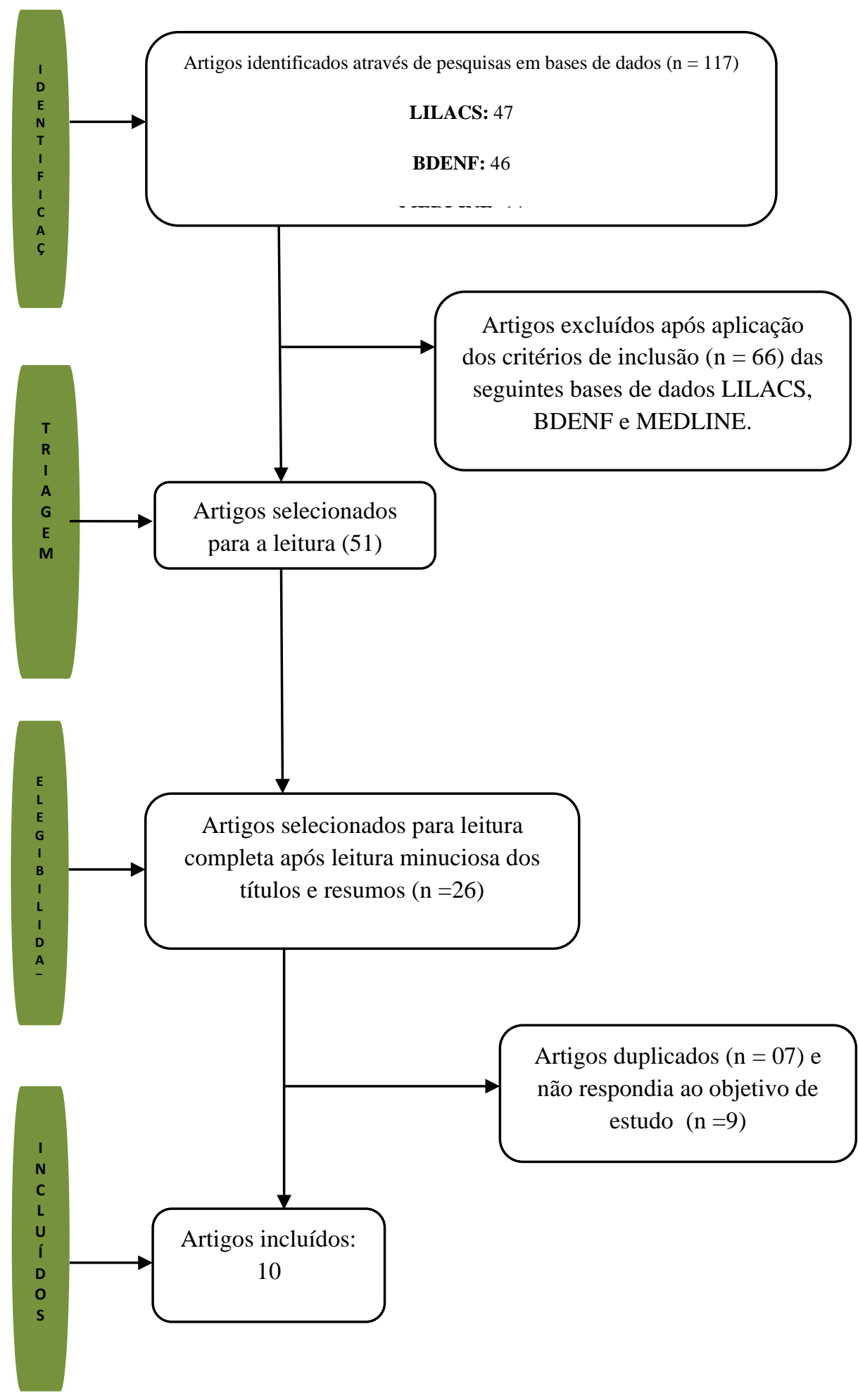

Fonte: Fluxograma de seleção de estudos primários. Teresina, Pi, Brasil (2021).

Como observado na figura anterior 10 artigos foram selecionados de acordo com os critérios de inclusão e exclusão respeitado a estratégia de busca utilizada para o desenvolvimento do trabalho.

Quadro 1 é apresentada a síntese da amostra analisada e consta as informações quanto à bases de dados, título, ano, idioma e tipo de estudo. 
Quadro 1. Perfil das produções ( $\mathrm{n}=10)$.

\begin{tabular}{|c|c|c|c|c|}
\hline $\begin{array}{l}\text { BASE DE } \\
\text { DADOS }\end{array}$ & TITULO & ANO & IDIOMA & TIPO DE ESTUDO \\
\hline SCIELO & $\begin{array}{l}\text { A saúde dos profissionais de saúde no enfrentamento da } \\
\text { pandemia de Covid-19 }\end{array}$ & 2020 & Português & Estudo transversal \\
\hline SCIELO & $\begin{array}{l}\text { O estresse e a saúde mental de profissionais da linha de } \\
\text { frente da COVID-19 em hospital geral }\end{array}$ & 2021 & Português & Análise transversal \\
\hline SCIELO & $\begin{array}{l}\text { Círculo de cultura virtual: promovendo a saúde de } \\
\text { enfermeirosno enfrentamento da covid-19 }\end{array}$ & 2021 & Português & $\begin{array}{l}\text { Relato de } \\
\text { experiência }\end{array}$ \\
\hline SCIELO & $\begin{array}{l}\text { Saúde mental da enfermagem no enfrentamento do } \\
\text { COVID- } 19 \text { em um hospital universitário regional }\end{array}$ & 2020 & Português & $\begin{array}{c}\text { Estudo de } \\
\text { observacional }\end{array}$ \\
\hline LILACS & $\begin{array}{l}\text { Investigação epidemiológica de Covid-19 relacionada ao } \\
\text { trabalho em trabalhadores de Saúde: experiência do } \\
\text { Cerest Salvador }\end{array}$ & 2021 & Português & Estudo observacional \\
\hline MEDLINE & COVID-19 and mental health: Self-care for nursing staff. & 2020 & Inglês & Estudo diagnóstico \\
\hline MEDLINE & $\begin{array}{l}\text { COVID-19: Nurses have responded, now it is time to } \\
\text { support them as we move forward. }\end{array}$ & 2020 & Inglês & Estudo diagnóstico \\
\hline MEDLINE & $\begin{array}{l}\text { The Psychological Change Process of Frontline Nurses } \\
\text { Caring for Patients with COVID-19 during Its Outbreak. }\end{array}$ & 2020 & Inglês & Estudo prognóstico \\
\hline MEDLINE & $\begin{array}{l}\text { A qualitative study on the psychological experience of } \\
\text { caregivers of COVID-19 patients. }\end{array}$ & 2020 & Inglês & Pesquisa qualitativa \\
\hline MEDLINE & $\begin{array}{l}\text { Guia de orientações para organização e fluxo nas } \\
\text { Unidades Básicas de Saúde em tempo de Coronavírus }\end{array}$ & 2020 & Português & Estudo observacional \\
\hline
\end{tabular}

Fonte: Biblioteca Virtual de Saúde (BVS).

Por se tratarem de estudos de variadas vertentes que buscaram analisar detalhadamente os desafios dos profissionais de enfermagem frente a pandemia do coronavírus sars-cov2 os parâmetros avaliados por cada estudo representam a posterior apresentação de resultados correlacionados aos seus principais achados de forma independente.

Como exposto na figura anterior, observa-se a maioria dos estudos com ano de publicação 2020 totalizando 7 artigos, apresentando apenas 3 estudos do ano de 2021. No que se refere aos idiomas empregados, não foram selecionados artigos no idioma espanhol, sendo a língua portuguesa a predominante, empregada em 6 dos artigos. Na abordagem metodológica são 3 artigos de estudo transversal, 2 observacionais, 1 relato de experiência, 2 estudos diagnósticos, 1 prognostico e 1 qualitativo. Vale ressaltar a diversidade de periódicos, em que houve o emprego maior na base de dados em Enfermagem MEDLINE com 5 publicações, seguida da Scientific Electronic Library (SCIELO), com 4 publicações e LILACS 1 estudo.

Já o quadro 2 aborda os resultados em evidência retirados de cada publicação após uma análise criteriosa, feita por dois revisores separadamente, e enviadas a um terceiro, a fim de responder o seguinte questionamento: "Quais as evidências científicas acerca dos desafios dos profissionais de enfermagem frente a pandemia pelo Coronavírus SARS-COV2?" 
Quadro 2. Quadro com os Resultados em evidência $(n=10)$.

\begin{tabular}{|c|c|c|}
\hline $\mathbf{N}^{\mathbf{o}}$ & Título & $\begin{array}{l}\text { Quais as evidências científicas acerca dos desafios dos profissionais de } \\
\text { enfermagem frente a pandemia pelo Coronavírus SARS-COV2, com destaque } \\
\text { para os aspectos que consideram relevantes sobre a temática? }\end{array}$ \\
\hline 01 & $\begin{array}{l}\text { A SAÚDE DOS } \\
\text { PROFISSIONAIS DE SAÚDE } \\
\text { NO ENFRENTAMENTO DA } \\
\text { PANDEMIA DE COVID-19 }\end{array}$ & $\begin{array}{l}\text { O estudo apontou que o principal fator que tem gerado afastamento do trabalho é a } \\
\text { contaminação, além do intenso sofrimento emocional que é expressado em transtorno } \\
\text { de ansiedade, distúrbios do sono, o medo de contaminar familiares e amigos e medo da } \\
\text { morte. O que evidencia parte dos problemas enfrentados pelos profissionais de saúde. }\end{array}$ \\
\hline 02 & $\begin{array}{l}\text { O ESTRESSE E A SAÚDE } \\
\text { MENTAL DE PROFISSIONAIS } \\
\text { DA LINHA DE FRENTE DA } \\
\text { COVID-19 EM HOSPITAL } \\
\text { GERAL }\end{array}$ & $\begin{array}{l}\text { Este estudo ao investigar os efeitos da atuação na linha de frente da Covid-19 a saúde } \\
\text { mental dos profissionais de saúde apresenta alto sofrimento psicossocial, stresse e } \\
\text { burnout, além de detectar um grande número de profissionais afastados de suas } \\
\text { atividades aborais. }\end{array}$ \\
\hline 03 & $\begin{array}{l}\text { CÍRCULO DE CULTURA } \\
\text { VIRTUAL: PROMOVENDO A } \\
\text { SAÚDE DE ENFERMEIROSNO } \\
\text { ENFRENTAMENTO DA } \\
\text { COVID-19MELLITUS. }\end{array}$ & $\begin{array}{l}\text { O estudo apontou que as enfermeiras se tornaram mais fortes após as várias } \\
\text { experiências que tiveram no seu ambiente de trabalho, com isso tornaram-se } \\
\text { empoderadas para assim encarar de frente todos os desafios que a pandemia trouxe. }\end{array}$ \\
\hline 04 & $\begin{array}{l}\text { SAÚDE MENTAL DA } \\
\text { ENFERMAGEM NO } \\
\text { ENFRENTAMENTO DO } \\
\text { COVID-19 EM UM HOSPITAL } \\
\text { UNIVERSITÁRIO REGIONAL }\end{array}$ & $\begin{array}{l}\text { Segundo este estudo a enfermagem diariamente precisa lidar com situações e fatores } \\
\text { estressantes, e no cenário atual frente a pandemia pela covid-19 houve um impacto } \\
\text { ainda maior na saúde mental dos enfermeiros. Por isso aponta a importância destes } \\
\text { profissionais buscarem o máximo de conhecimento e ajuda para assim lidarem terem } \\
\text { uma qualidade de vida e saúde melhor. }\end{array}$ \\
\hline 05 & $\begin{array}{c}\text { INVESTIGAÇÃO } \\
\text { EPIDEMIOLÓGICA DE } \\
\text { COVID-19 RELACIONADA AO } \\
\text { TRABALHO EM } \\
\text { TRABALHADORES DE } \\
\text { SAÚDE: EXPERIÊNCIA DO } \\
\text { CEREST SALVADOR }\end{array}$ & $\begin{array}{l}\text { O estudo demonstrou que através da realização de investigação de casos de Covid-19 } \\
\text { relacionados ao ambiente de trabalho, por iniciativa do Cerest Salvador, ofertou uma } \\
\text { grande possibilidade para o diagnóstico situacional da Covid- } 19 \text { entre os profissionais } \\
\text { de enfermagem no município, reforçando a importância dos ambientes e processos de } \\
\text { trabalho no aumento do número casos da doença. }\end{array}$ \\
\hline 06 & $\begin{array}{l}\text { COVID-19 AND MENTAL } \\
\text { HEALTH: SELF-CARE FOR } \\
\text { NURSING STAFF. }\end{array}$ & $\begin{array}{l}\text { Este estudo enfatiza a seriedade dessa doença de propagação tão rápida que e a Covid- } \\
19 \text { e suas repercussões a curto e longo prazo na vida dos profissionais de saúde. Com } \\
\text { isso tora evidente a necessidade de apoio, estratégias de autocuidado entre outras } \\
\text { estratégias para reestabelecer sua saúde mental e física. }\end{array}$ \\
\hline 07 & $\begin{array}{l}\text { COVID-19: NURSES HAVE } \\
\text { RESPONDED, NOW IT IS } \\
\text { TIME TO SUPPORT THEM AS } \\
\text { WE MOVE FORWARD. }\end{array}$ & $\begin{array}{l}\text { O estudo detectou o ardo mental e físico que a Covid-19 impôs sobre o profissional } \\
\text { enfermeiro à medida que se colocam em risco a linha de frente para proteger os outros, } \\
\text { tornando clara a necessidade da criação de estratégias que ajudem no fortalecimento e } \\
\text { na cura emocional destes profissionais. }\end{array}$ \\
\hline 08 & $\begin{array}{l}\text { THE PSYCHOLOGICAL } \\
\text { CHANGE PROCESS OF } \\
\text { FRONTLINE NURSES CARING } \\
\text { FOR PATIENTS WITH COVID- } \\
19 \text { DURING ITS OUTBREAK. }\end{array}$ & $\begin{array}{l}\text { Ao investigar o processo de mudança psicológica de enfermeiros atuantes na linha de } \\
\text { frente o estudo demonstrou a necessidade das lideranças de enfermagem } \\
\text { implementarem ações que promovam saúde mental aos enfermeiros atuantes neste } \\
\text { período tão crítico. }\end{array}$ \\
\hline 09 & $\begin{array}{l}\text { A QUALITATIVE STUDY ON } \\
\text { THE PSYCHOLOGICAL } \\
\text { EXPERIENCE OF } \\
\text { CAREGIVERS OF COVID-19 } \\
\text { PATIENTS. }\end{array}$ & $\begin{array}{l}\text { O estudo aponta que durante a pandemia pelo coronavírus um misto de emoções } \\
\text { positivas e negativas se entrelaçaram, pois de início as emoções negativas } \\
\text { predominavam, já as positivas foram aparecendo de forma gradativa, demonstraram } \\
\text { que estilos de autocuidado desempenharam um papel importante na manutenção da } \\
\text { saúde mental dos profissionais. }\end{array}$ \\
\hline 10 & $\begin{array}{l}\text { GUIA DE ORIENTAÇÕES } \\
\text { PARA ORGANIZAÇÃO E } \\
\text { FLUXO NAS UNIDADES } \\
\text { BÁSICAS DE SAÚDE EM } \\
\text { TEMPO DE CORONAVÍRUS }\end{array}$ & $\begin{array}{l}\text { O estudo objetivou ressaltar um pouco sobre as formas de organização, prevenção e } \\
\text { controle e prestar o devido auxílio para os gestores e profissionais a realizarem } \\
\text { escolhas eficientes com relação ao novo coronavírus. Segundo os professores tudo o } \\
\text { que foi orientado através do guia foi baseados no que se sabe até o momento por se } \\
\text { tratar de m vírus novo. }\end{array}$ \\
\hline
\end{tabular}


Analisou-se que todos os 10 artigos trabalhavam desafios dos profissionais de enfermagem frente a pandemia do coronavírus sars-cov2, demonstrando a importância do cuidado e manutenção da saúde desses profissionais.

\section{Discussão}

Para Horta et al., (2021) os desafios psicológicos enfrentados pelos profissionais são na maioria das vezes reflexo da sobre carga trabalhista onde a priorização por repouso e intervalos podem ser associados para uma melhora no desenvolvimento do trabalho dos mesmos. Já para Miranda et al., (2020) é compreensível que, devido à pandemia do COVID19, a carga de trabalho seja excessiva e a alta especificidade de transmissão do vírus tenha proporcionado um momento ímpar na assistência de enfermagem. E o manuseio de equipamentos de proteção específicos.

O uso de EPI que também, devido à emergência e em alguns casos, o colapso ocasionado por ela, pode apresentar-se inferior a demanda necessária para atendimento adequado e seguro. É de suma importância o atentar-se à classificação das atividades executadas nos ambientes de saúde. (Teixeira et al., 2020). Adoecimento no decorrer dos meses, afastamento por motivos psicológicos, diminuição do quadro de colaboradores por afastamento do grupo de risco, sobrecarga no trabalho, estresse, insegurança nos atendimentos nas situações de maior gravidade por falta de capacitação, apreensão em relação a paramentarão e desparamentação (Reis et al., 2020).

Em um surto epidêmico, as emoções positivas e negativas das enfermeiras da linha de frente se entrelaçaram e coexistiram. No estágio inicial, as emoções negativas dominam e as emoções positivas aparecem gradualmente. Os métodos de autocuidado e o crescimento psicológico desempenham um papel importante na manutenção da saúde mental dos enfermeiros (Sun et al., 2020).

Para Souza et al., (2021) deve ser dada prioridade à identificação de riscos e ao planejamento de intervenções para reduzir os danos à saúde mental dos profissionais que cuidam de pessoas que cuidam ou são diagnosticadas com COVID-19. Os profissionais de saúde e suporte devem usar equipamento de proteção individual antes de tocar nas roupas pessoais ou roupas de cama de usuários suspeitos de ter Covid-19. Tirar roupas sujas requer agitação e manuseio mínimos para evitar a propagação do vírus, portanto, elas devem ser embaladas em um saco plástico exclusivo marcado (Moreira et al,. 2020). Visando promover mudanças no comportamento em relação às medidas preventivas como, por exemplo, o uso obrigatório de máscaras de proteção nasal e oral em locais públicos e assim reduzir os possíveis contágios e consequentemente redução de hospitalizações e infecção dos trabalhadores em saúde que prestam assistência direta aos pacientes, como por exemplo a equipe de enfermagem, contudo diminuindo os conflitos e desafios dessa categoria vivendo hoje em meio à tantas incertezas e medos e que historicamente sempre atuou em momentos de crise, como protagonistas atuantes na linha de frente (Oliveira, et al., 2020, Clancy, et al., 2020).

De acordo com Mendes et al., (2021) a pandemia Covid-19 comprova a vulnerabilidade dos trabalhadores da saúde, seja pelas más condições de trabalho, pela falta de equipamentos de proteção coletiva e individual, ou mesmo pelas relações de trabalho instáveis. Ressalta Bosco et al., (2020) identificar a relação com o trabalho e como ela funciona na disseminação da Covid-19 é essencial para intervir e responder à pandemia no local de trabalho. Devido à pressão interna (medo, incapacidade de enfrentar a dor, falta de conhecimento) ou externa (hierarquia, comunicação e questões organizacionais), o sofrimento moral pode dificultar a tomada de decisões e às vezes até levar à insegurança e incerteza durante uma pandemia, levando a este colapso emocional profissional (Santos et al., 2021).

Vulnerabilidade a contaminação pela assistência prestada direta ao paciente, condições de trabalho precário, subdimensionamento de trabalho (Collins et al., 2020). O processo de mudança psicológica do enfermeiro de primeira linha inclui três etapas: inicial, intermediária e subsequente. As características psicológicas de cada período são ambivalência, 
exaustão emocional e energia. Os enfermeiros líderes são a principal força para promover a adaptação psicológica dos enfermeiros da linha da frente (Zhang et al., 2020).

\section{Conclusão}

Com base nos estudos analisados concluiu-se que os profissionais de enfermagem sempre foram desafiados ao longo do tempo e com a chegada da pandemia pelo Coronavírus SARS-COV2 esses desafios tomaram proporções assustadoras e desafiadoras. Além da desvalorização salarial, a sobrecarga de trabalho, a escassez de EPI's, infraestruturas e locais de trabalho inadequados, esses profissionais passaram a conviver com medo de se contaminar com o vírus ou levar contaminação para seus familiares e isso gerou problemas de saúde para essa classe trabalhadora.

Enquanto trabalhadores da saúde, a equipe de enfermagem são os profissionais que passam mais tempo com os pacientes e prestam assistência e cuidados direto aos mesmos e isso vem tornando-os mais vulneráveis a contaminação pelo coronavírus e consequentemente há um número crescente de óbitos entre esses colaboradores.

Em virtude da magnitude dos problemas de saúde vivenciados atualmente pela presença do Vírus SARS-COV2 em todos os ambientes, é o momento oportuno para o mundo inteiro olhar para esses profissionais e repensar sobre a importância que essa categoria tem para a humanidade, pois mesmo com todos os desafios enfrentados, todos os medos superados, a ausência da família, mesmo na exaustão física e mental, a enfermagem segue trabalhando na linha frente de forma contínua, com apenas um objetivo que é salvar vidas alheias que são riquezas incalculáveis.

Diante do estudado sugere-se que haja o desenvolvimento de estudos com a temática em questão para que o debate a respeito destes desafios para assim ter embasamento para desenvolver estratégias que melhorem a qualidade da saúde física e emocional dos profissionais da saúde.

\section{Referências}

Santos, T. A., Santos, H. S., Moraes, M. A., Mussi, F. C. (2020). Comiittee to Coping with COVID-19 in Bahia. Rev Bras Enferm. 1-5

A, F. Bahiano, M. A., Nakano, T. C., Silva, B. F. P., Viti, L. S. (2020). COVID-19 e saúde mental: a emergência do cuidado. Estud. Psicol. 1-14.

Rhonda Collins. (2020). COVID-19: Nurses have responded, now it is time to support them as we move forward. Healthcare Management Forum. 190-194.

Dal'Bosco, E. B., Floriano, L. S. M., Skupien, S. V., Arcaro, G., Martins, A. R., Anselmo, A. C. C. (2020). Saúde mental da enfermagem no enfrentamento do COVID-19 em um hospital universitário regional. Rev. Bras. Enferm. 1-7

Duarte, M. L. C., Silva, D. G., Bagatini, M. M. C. (2020). Enfermagem e saúde mental: uma reflexão em meio à pandemia de coronavírus. Rev Gaúcha Enferm. 1-6.

Lima, L. S., Bessa, M. M., Silva, S. W. S., Moura, K. M. Souza, J. O., Freitas, R. J. M. (2021). Processo de enfermagem para pacientes com manifestações respiratórias da COVID-19. Rev. enferm. UFPE on line. 1-10.

Mendes, T. T. M., Ribeiro, A. P. C., Andrade, C. A. S., Bastos, P. K. F., Pádua, P. D. (2021). Investigação epidemiológica de Covid-19 relacionada ao trabalho em trabalhadores de Saúde: experiência do Cerest Salvador. Revista Baiana de Saúde Pública. 254-266.

Miranda, F. M. D., Santana, L. L., Pizzolato, A. C., Sarques, L. M. M. (2020). Rev Cogitare enferm. 1-8.

Moreira, A. D. Freitas, G. L., Montenegro, L. C. Lachtim, S. A. F. (2020). Guia de orientações para organização e fluxo nas Unidades Básicas de Saúde em tempo de Coronavírus. Universidade Federal de Minas Gerais. 1-25.

Oliveira, Adriana Cristina. (2020). Desafios da enfermagem frente ao enfrentamento da pandemia da COVID19. Rev Min Enferm. 1-8.

Reis, L. M., Lago, P. Carvalho, A. H. S., Nobre, V. N. N., Guimarães, A. P. R. (2020). Atuação da enfermagem no cenário da pandemia COVID-19. Revista Nursing. 4765-4772.

Santos, K. M. R., Galvão, M. H. R., Gomes, S. M., Souza, T. A., Medeiros, A. A., Barbosa, I. R. (2021). Esc Anna Nery. 1-15.

Souza, J. B., Vendruscolo, Carine., Bitencourt, J. V. O., Brum, C. N., Luzardo, A. R. (2021). Círculo de cultura virtual: promovendo a saúde de enfermeiros no enfrentamento da covid-19. Rev. Gaúcha Enferm. 1-7. 
Rolim, A. J., Oliveira, A. R., Batista, E. C. (2020). Manejo da Ansiedade no Enfrentamento da Covid-19. Rev Enfermagem e Saúde Coletiva. 64-74.

Teixeira, C. F. S., Soares, C. M., Souza, E. A., Lisboa, E. S., Pinto, I. C. M., Andrade, L. R., Espiridião, M. A. (2020). A saúde dos profissionais de saúde no enfrentamento da pandemia de Covid-19. Ciênc. saúde coletiva. 3465- 3474.

Zhang, Yan. (2020). The Psychological Change Process of Frontline Nurses Caring for Patients with COVID-19 during Its Outbreak. Issues Ment Health Nurs. 525-530.

Ramos, A. R., Bottega, C. G., Petersen, L. L., Rollo, R. M., Marchioro, M. K., Rocha, C. M. F. (2021). COVID-19: repercussões para enfermagem, estruturação e resolutividade de sistemas nacionais de saúde. Rev Gaúcha Enferm. 1-14.

Soares, S. S. S., Souza, N. V. D., Carvalho, E. C., Varella, T. C. M., Andrade, K. B. S., Pereira, S. R. M., Costa, C. C. P. (2020). De Cuidador a Paciente: na pandemia da COVID-19, quem defende e cuida da enfermagem brasileira? Esc. Ana Nery. 1-7.

Horta, R. L., Camargo, E. G., Barbosa, M. L. L., Lantin, P. J. S., Sette, T. G., Lucini, T. C. G., Silveira, A. F., Zanini, L., Lutzky, B. A. (2021). O estresse e a saúde mental de profissionais da linha de frente da COVID-19 em hospital geral. J. bras. psiquiatr. 30-38.

Lima, L. S., Bessa, Marcelino M. M., Silva, S. W. S., Moura, K. M., Souza, J. O., Freitas, R. J. M. (2021). Processo de enfermagem para pacientes com manifestações respiratórias da COVID-19. Rev. enferm. UFPE on line ; 15(1): (1-10).

Minayo, M. C. S., Freire, N. P. (2020). Pandemia exacerba desigualdades na Saúde. Rev Ciência e Saúde Coletiva. 1-2.

Quadros, A., Fernandes, M. T. C., Araujo, B. R., Caragnato, R. C. A. (2020). Desafios da enfermagem brasileira no combate ao COVID-19. Doente foco (Brasília). 78-83

Santos, V. C., Persegona, M. F. M., Souza, E. F., Almeida, W. C., Filete, M., Silva, M. C. N. (2020). Comitê gestor de crise do coronavírus no âmbito do cofen. Rev Enferm. Foco. 6-10.

Ercole, F. F., Melo, L. S., Alcoforado, C. L. G. C. (2020). Revisão integrativa versus revisão sistemática. Revista Mineira de Enfermagem. 9-11. 